Thomas, M.B \& Read, A.F. 2007. Can fungal biopesticides control malaria? Nature Microbiology Reviews 5: 377-383.

\title{
Can fungal biopesticides control malaria?
}

\author{
Matt B. Thomas and Andrew F. Read
}

Matt Thomas - is a population ecologist specialising in biological pest control. His group has experience studying a range of biocontrol problems in temperate and tropical settings including several years working as part of a research programme to develop a biopesticide for control of locusts and grasshoppers in Africa. He is currently co-chair of an international consortium researching novel use of fungi for control of mosquitoes and malaria in Africa.

Andrew Read - is an evolutionary ecologist specialising in host-parasite interactions. He is particularly interested in the evolution of pathogen traits of medical and veterinary importance, including infectiousness, virulence, drug resistance and vaccine escape. Most of his work has involved malaria, but his group has also worked on viruses, bacteria, trypanosomes and nematodes. A major theme is trying to understand how public and animal health interventions shape pathogen evolution, and how to avoid evolutionary mismanagement of infectious disease.

Abstract | Recent research has raised the prospect of using insect fungal pathogens for the control of vector-borne diseases such as malaria. In the past, microbial control of insect pests in both medical and agricultural sectors has generally had limited success. We propose that it may now be possible to produce a cheap, safe and green tool for the control of malaria which, in contrast to most chemical insecticides, will not eventually be rendered useless by resistance evolution. Realising this potential will require lateral thinking by biologists, technologists and development agencies. 
Thomas, M.B \& Read, A.F. 2007. Can fungal biopesticides control malaria? Nature Microbiology Reviews 5: 377-383.

A key component of integrated control of vector-borne diseases such as malaria and dengue is the use of insecticides that target the insect vectors. However, the utility of insecticides is being undermined by problems of insecticide resistance, environmental contamination and risks to human health ${ }^{1-3}$. Therefore alternative approaches are required $^{2}$. Biocontrol using biopesticides that are based on naturally occurring microbial pathogens is one such method.

Insects can be infected by bacterial, viral, protozoan and fungal pathogens. Of these, fungal entomopathogens are perhaps the best-suited for development as biopesticides because they do not require ingestion by the host. Instead, fungi infect by external contact with the host (see FIG 1). The time taken to kill the host following infection varies from 2-5 days to days or weeks, depending on the particular hostpathogen combination and environmental conditions ${ }^{4}$.

Few biopesticide products have been widely used, in spite of their potential. Indeed, on a global scale, penetration of biocontrol technology into the pesticide market has been minimal; less than $2 \%$ of global pest control sales (US\$30b annually) comprise biocontrol and $>70 \%$ of this small proportion are biopesticide products that are based on the crystal toxin-forming bacterium Bacillus thuringiensis ${ }^{5,6}$.

This failure to adopt biocontrol strategies raises a fundamental question: is the success of microbial biocontrol limited by inadequate technology, unfavourable economics or a complex interplay of several factors? In this article we draw on recent advances in the development of biopesticides based on entomopathogenic fungi to explore this question. We use insights from the recent successful development of biopesticides for the control of locusts and grasshoppers to examine the potential for 
Thomas, M.B \& Read, A.F. 2007. Can fungal biopesticides control malaria? Nature Microbiology Reviews 5: 377-383.

development of a biopesticide to infect mosquitoes in resting and breeding sites in residential settings.

\section{Biopesticides for locusts and grasshoppers}

In 1989, in response to concerns over the environmental and human health consequences of extensive chemical applications against locusts and grasshoppers in Africa, the international donor community supported the initiation of a collaborative research programme to develop a more sustainable, biological pesticide for locust and grasshopper control. The programme, named LUBILOSA (Lutte Biologique contre les Locustes et les Sauteriaux), was founded on preliminary research that had identified a virulent strain of the entomopathogenic fungus, Metarhizium anisopliae var. acridum, and had revealed how formulation of fungal spores in oil could enable infection in the very low relative humidity conditions ${ }^{7,8,9}$ (see FIG 2). The end product was Green Muscle ${ }^{\circledR}$, which has now been registered in several countries including South Africa, Zambia, Namibia, Sudan, Mozambique and much of French West Africa. Since LUBILOSA finished in 2001, several projects have continued to evaluate the impact of Green Muscle ${ }^{\circledR}$ on locust and grasshopper species in Africa and Europe, to optimize its usage 9

In 1993, the Commonwealth Scientific and Industrial Research Organisation (CSIRO) collaborated with LUBILOSA to develop a biopesticide (based on an Australian strain of the same subspecies used in Africa) for use against locusts and grasshoppers in Australia $^{10,11}$. This resulted in Green Guard®, which was registered in $2005^{11}$. Green 
Thomas, M.B \& Read, A.F. 2007. Can fungal biopesticides control malaria? Nature Microbiology Reviews 5: $377-383$

Guard® now forms an integral part of locust control operations in Australia, with a steadily increasing market share as the technology becomes established ${ }^{11,12}$.

The production of Green Muscle ${ }^{\circledR}$ and Green Guard ${ }^{\circledR}$ show that effective biopesticide products based on entomopathogenic fungi can be developed. LUBILOSA cost $\sim$ US $\$ 17$ million, which compares well with the estimated US\$70-100 million that is required to develop a new synthetic pesticide compound ${ }^{9}$. Although there are some cheaper products available, Green Muscle $₫$ and Green Guard $₫$ are price competitive with most established insecticides. Importantly, of all the products for locust and grasshopper control, they have the lowest environmental impact and can be used near water courses, organic crops and conservations areas, satisfying the demand for more environmentally sustainable technologies.

More generally, the locust biopesticide programmes have advanced our knowledge in a range of areas such as isolate screening, formulation, mass production, quality control, storage, application, environmental impact, safety testing and hostpathogen ecology. These technical advances have been accompanied by developments in capacity in areas such as commercial production and distribution, product registration and extension to end-users. Overall, such developments provide a solid foundation for the development of fungus-based biopesticides for use in integrated strategies for control of diseases such as malaria (and also dengue and filariasis).

\section{Proof of concept for malaria control}

Several studies have investigated the use of microbial biocontrol to kill mosquitoes (for a review, see REFS 13-15). Typically, virulent pathogens have been isolated, with the aim 
Thomas, M.B \& Read, A.F. 2007. Can fungal biopesticides control malaria? Nature Microbiology Reviews 5: 377-383.

of developing biopesticides to kill mosquito larvae ${ }^{13,14,16}$. More recently several studies have highlighted the potential use of fungal pathogens to kill adult mosquitoes ${ }^{17-20}$. The common approach behind these studies is to infect insects by exposure to oil-formulated fungal spores that have been applied to surfaces on which adult mosquitoes rest after blood meals.

Initial laboratory-based bioassays revealed that mosquitoes were readily infected by exposure to entomopathogenic fungi and that some fungal isolates caused $100 \%$ mortality of adult Anopheles and Culex spp. in 7-14 days, depending on dose, formulation and fungal $\operatorname{strain}^{17-19}$. Further studies used a rodent malaria model to examine the effect of fungal infection on malaria transmission potential ${ }^{19}$ (see FIG 3). The results indicated an 80-fold reduction in the number of mosquitoes able to transmit malaria following exposure of the insects to the fungal pathogen. This reduction resulted from two complementary effects of fungal infection. First, fungal infection caused high levels of mosquito mortality by day 14 after blood feeding (when sporozoites are present in the mosquito mouthparts). Moreover, the daily mortality rate of mosquitoes infected with both fungus and malaria increased compared with insects infected with just the fungus from day 11. Second, significantly fewer surviving mosquitoes had sporozoites in their mouthparts compared with control mosquitoes infected with malaria alone, indicating a negative effect of fungal infection on survivorship/development of the malaria parasite inside the mosquito. In addition, fungus-infected mosquitoes were less likely to bloodfeed (FIG 3), further reducing transmission potential ${ }^{19,21}$. Finally, a small-scale study in village houses in Tanzania confirmed the feasibility of infecting mosquitoes with virulent fungi under field conditions in Africa ${ }^{20}$. This investigation used a relatively low dose of 
Thomas, M.B \& Read, A.F. 2007. Can fungal biopesticides control malaria? Nature Microbiology Reviews 5: $377-383$.

an experimental formulation applied over a small surface area, but still showed that $34 \%$ of mosquitoes collected from targeted village houses were infected with fungi. Simple epidemiological models predict that even this relatively low level of infection would result in a $75 \%$ reduction in Entomological Inoculation Rate (EIR) at this field $\operatorname{site}^{20}$. The study used fungus-treated black cloths that were pinned to the ceilings of dwellings. These cloths could be repeatedly treated with spores at relatively little cost or inconvenience.

These studies highlight the potential of fungal biopesticides to substantially reduce mosquito vectorial capacity using currently available technology. However, the literature is littered with examples of promising microbial agents and candidate biopesticide technologies yet, as evidenced by the very limited penetration of the chemical pesticide market, very few realize this potential. So we consider next some of the features that represent both the strengths and weaknesses of the fungal biopesticide approach. We draw on lessons from the locust biopesticide research and consider specific aspects relating to biopesticide control of malaria. As such we do not consider more generic, albeit important, $R \& D$ issues such as optimization of production, delivery systems, field testing, or safety issues (for a discussion of the latter see REFS 22-24).

\section{Disease pathology and biopesticides}

A crucial factor for the successful transmission of malaria is the longevity of the mosquito compared with the approximately two week parasite incubation period ${ }^{25,26}$. Even small reductions in adult mosquito longevity after an infective blood meal can have a large impact on malaria dynamics. Thus, unlike most other insect control problems it is 
Thomas, M.B \& Read, A.F. 2007. Can fungal biopesticides control malaria? Nature Microbiology Reviews 5: 377-383.

not necessary to rapidly kill the mosquito with a virulent pathogen. Moreover, emphasis on the 'pesticidal' properties of entomopathogens overlooks their potential to influence insect behaviour and fitness in subtle ways that may negatively impact malaria transmission, without necessarily reducing vector density. For example, numerous insectpathogen studies indicate the potential for sub- or pre-lethal pathogen effects. Locusts infected with entomopathogenic fungi have altered feeding behaviour, fat body accumulation, development rate, fecundity, mobility and predator escape responses before death ${ }^{27-31}$. Similarly, preliminary research indicates that infected mosquitoes may have a reduced propensity to feed prior to death ${ }^{19,21}$ and there is evidence that fungal pathogens can affect not only the mosquito, but also the survivorship of malaria within the mosquito ${ }^{19}$. The mechanisms that underlie this anti-malaria effect are unknown but might include alterations in host nutritional balance leading to resource competition, upregulation of immune responses, or production of secondary metabolites in the haemolymph.

The deleterious effects of sub-lethal pathogens on the capacity of insects to function as vectors of disease has been virtually ignored although sub-lethal effects are the most common outcome of infection. Exploiting sub-lethal effects of pathogens could present new opportunities for development of biopesticides.

\section{Evolution of resistance}

Anopheles mosquitoes have proved adept at evolving resistance to chemical insecticides ${ }^{32-34}$. Indeed, resistance to insecticides has appeared in the major insect vectors from every genus, with examples of resistance to every chemical class of 
Thomas, M.B \& Read, A.F. 2007. Can fungal biopesticides control malaria? Nature Microbiology Reviews 5: 377-383.

insecticide $^{35}$. Biopesticide control would be similarly unsustainable if the widespread use of fungal entomopathogens provided a selective pressure that resulted in evolution of fungal resistance mechanisms in mosquitoes ${ }^{22,36}$.

Little is known about genetic variation in fungal susceptibility among Anopheles populations. All mosquitoes may be fully susceptible (we can find no records of complete resistance against fungal pathogens in any insect). However, there is evidence for genetic variation in susceptibility (time to death) to entomopathogenic fungi in aphids $^{37,38}$ and Drosophila ${ }^{39}$, as well as environmentally ${ }^{40}$ and behaviourally-mediated host responses that alter effective resistance ${ }^{4,41,42}$. Moreover in the long history of malaria control, resistance to all interventions has eventually evolved, even in the absence of pre-existing resistance. If biopesticides are to avoid the depressing fate of so many other malaria control measures, we need to maximise the reduction in malaria transmission without imposing strong selection on vector populations. There are several reasons for thinking that this might be achievable with a fungal biopesticide.

First, the negative effects of pathogenic fungi on the mosquito host occur relatively late in the lifecycle of the mosquito. Fungal-induced mosquito mortality and reduced propensity to blood feed occur after most mosquitoes in natural populations have already died (FIG 4). It is well known in the context of the evolution of ageing that beneficial mutations acting late in life are subject to weak selection because they confer fitness benefits after the majority of individuals have anyway ceased reproducing ${ }^{43,44}$. Thus, even if Anopheles could develop resistance to fungi, biopesticides might impose only weak selection for that resistance. Such reductions in selection pressure could translate into decades more of effective use of a product. Moreover, there may actually 
Thomas, M.B \& Read, A.F. 2007. Can fungal biopesticides control malaria? Nature Microbiology Reviews 5: 377-383.

be no selection for resistance. If the possession of fungal resistance mechanisms entails metabolic costs, all individuals in a population would pay the price for a benefit experienced only by a few. Indeed, while it might be tempting to deploy more virulent isolates that either kill insects more quickly, or kill insects at a constant daily rate, this capacity for killing would need to be balanced against potentially sharp increases in selection pressure to evolve resistance.

This argument is subject to a couple of corollaries. Slow speed of kill potentially increases the level of biopesticide coverage necessary to impact on malaria transmission because the slower the speed of kill, the greater the need for the mosquito to become infected at the first or second blood feed. This will require that a high proportion of houses are treated and that there is a high probability of infection per feed. It might also be that fungal pathogens could place an evolutionary pressure on the malaria parasite to produce sporozoites before the fungus kills the host ${ }^{36}$. However, given that natural mosquito survival is so low, there must already be strong selection for more rapid development. There must, therefore, be very substantial fitness costs associated with shorter incubation periods. Even if fungal biopesticides did tip the balance in favour of more rapid development, it is difficult to assess the overall effect on human disease burdens of any such evolution without knowing what these fitness costs are.

A second reason for thinking that fungal biopesticides would not be undermined by mosquito resistance is the possibility that fungal infection has a direct anti-malarial effect, reducing the prevalence of sporozoites. It would be highly desirable to isolate fungal strains that had an increased propensity to reduce mosquito infectiousness, since this effect of the pathogen does not result in selection for fungal resistance in mosquitoes. 
Thomas, M.B \& Read, A.F. 2007. Can fungal biopesticides control malaria? Nature Microbiology Reviews 5: 377-383.

Indeed, some fungal isolates can reduce sporozoite prevalence without causing any mosquito mortality (Blanford, Read and Thomas unpubl data); this effect could in principle be enhanced by paratransgenesis (Box). Similar to above, however, products relying only on these anti-malarial effects might in the long run suffer from the evolution of resistant malaria parasites.

A third reason for thinking that biopesticides could be evolution-proof is that, mosquitoes infected with malaria parasites are more likely to die following fungal infection than mosquitoes that are not infected with the parasite (FIG 3). Malariainfected mosquitoes normally comprise less than $10 \%$ of the insect population. If the main effect of a fungal isolate was to reduce the fitness of malaria-infected mosquitoes (rather than any mosquito), this should reduce selection pressure for fungal resistance across the mosquito population overall, and may even select for increased malaria refractoriness ${ }^{45}$. Again, this would reduce malaria transmission without imposing a selection for fungal resistance.

Even if anti-fungal resistance is unlikely to emerge in response to biopesticide use, it would still be extremely interesting to understand mechanisms of fungal resistance in mosquitoes. For instance, are any resistance mechanisms isolate- or strain-specific? If they are, combinations of strains could be used in single biopesticide formulations to minimise further the risk for resistance evolution. In any case, because of the very different nature of fungal infection and the resultant insect immune response, it seems extremely unlikely that anti-fungal resistance would be related to 'metabolic' or 'knockdown' insecticide resistance mechanisms, so it should be possible to use biopesticides in localities in which evolution has rendered chemical insecticides obsolete. 
Thomas, M.B \& Read, A.F. 2007. Can fungal biopesticides control malaria? Nature Microbiology Reviews 5: 377-383.

\section{Formulation and application}

Application of spores inside houses, where many malaria vector species prefer to bloodfeed and rest, optimises the likelihood of fungus contact and infection. However, persistence of the fungal pathogens on treated surfaces has been identified as a key factor that will determine ultimate viability of the biopesticide approach ${ }^{46}$.

The active ingredient of a biopesticide is a living organism and so there will be biological limits to persistence. We must not expect that a biopesticide can, or necessarily should, have persistence characteristics similar to, for example, the long lasting insecticide treated nets, which can remain effective for several years ${ }^{47}$.

Preliminary studies indicate that viable spores can be recovered from treated surfaces after 3 months but that the percentage infection of mosquitoes exposed to these surfaces at this time is very low (Blanford, Read and Thomas unpubl. data). However, studies on of the use of fungal pathogens to control Tsetse fly (Glossina fuscipes) in Kenya indicated that spores retained their viability for 31 days in the field, and efficacy against G. fuscipes was not affected ${ }^{48}$. Moreover studies on spore storage indicate that fungal spores can remain viable for more than 12 months depending on prevailing temperature and humidity ${ }^{49,50}$. Thus, there is scope for achieving long-term infectivity but there is little understanding of the variation in infective half life between different fungal strains and how much persistence is determined by biological variation, versus factors such as dose and formulation under different environmental conditions. A fungal isolate that is only moderately pathogenic but that persists and remains infectious, could ultimately be more useful as a biopesticide than an isolate that is very virulent but 
Thomas, M.B \& Read, A.F. 2007. Can fungal biopesticides control malaria? Nature Microbiology Reviews 5: 377-383.

requires reapplication every two weeks. Similarly, an isolate that is easy to mass produce could prove more effective (both in terms of economics and impact) than an isolate that is more virulent but difficult to produce in operational quantities. The amenability of candidate microbial agents for commercial development has been identified as a critical factor in determining biopesticide success but is rarely considered as a criterion in isolate selection $^{51}$.

One area where there is substantial scope for maximising infectivity and persistence is through formulation. There is generally little specialist research on formulation of microbial agents ${ }^{52}$. However, the agrochemical, pharmaceutical and food processing industries have considerable expertise in producing formulations that enhance shelf life, protect products from decay and UV radiation and enable targeted or slow release of an active ingredient. The novel application of these established technologies could revolutionise biopesticide use ${ }^{52}$.

\section{Technology transfer and implementation}

In Australia, Green Guard ${ }^{\circledR}$ was used to treat $>60,000$ ha of locust infestations during the 2005-6 season ${ }^{12}$. Adoption of Green Muscle ${ }^{\circledR}$ in Africa, on the other hand, has been much more patchy. There are several factors contributing to the contrasting situations on the two continents, including differences in socio-economics, capacity, socio-political complexities and government and donor commitment ${ }^{9,53}$. The important insight, however, is that successful implementation and adoption require more than just technological innovation. 
Thomas, M.B \& Read, A.F. 2007. Can fungal biopesticides control malaria? Nature Microbiology Reviews 5: 377-383.

Studies on the demand for malaria control interventions indicate correlations between willingness to pay and socio-economic status ${ }^{54}$ and potentially very low threshold costs for deriving net benefit from control technologies ${ }^{55}$. Based on the current costs of products such as Green Muscle $\AA$ and Green Guard ${ }^{\circledR}$, and the experimental dose rates used in initial evaluation of fungi for control of malaria ${ }^{19}$, we estimate it would cost approximately US\$ 0.01 for enough biopesticide product to treat $1 \mathrm{~m}^{2}$. This is an encouraging figure, although it does not include labour costs, or the cost of cloth or netting for impregnation etc. and so cost will still be a major factor, including the question of who pays for the biopesticide. Both locust control biopesticides ultimately followed a public-private partnership model engaging small-to-medium-scale commercial companies to produce and distribute the products at national or regional levels. However, although requiring good quality control ${ }^{56,57}$ the methodologies for mass production are inherently 'low-tech' (see FIG 2). Local- (or even village-) scale production of biopesticides might be feasible, which would contribute towards ownership and acceptance of the technology at the community level. Such 'bottom-up' approaches are impossible with chemical insecticides but evidence suggests that control programmes are most successful when there is good cooperation owing to education, training and community involvement in implementation ${ }^{58}$. This need for cooperation identifies an important role for participatory approaches with end-users engaged early in the development process; something which is now recognised in the WHO policy for integrated vector management ${ }^{59}$.

Moreover, it is also important to match use (and user expectation) with product specification. Areas differ substantially in the seasonal incidence of malaria and their 
Thomas, M.B \& Read, A.F. 2007. Can fungal biopesticides control malaria? Nature Microbiology Reviews 5: 377-383.

epidemic versus endemic status. In some settings, 2 or 3 treatments of even a shortpersistence product could provide affordable, year-round control. Other settings might require repeated monthly applications which could prove prohibitive, depending on capacity and socio-economic context. An alternative a strategic approach could be to use a biopesticide over restricted temporal or spatial scales to disrupt cycles of resistance evolution and increase the durability of existing chemical interventions. This would represent a highly innovative application of biocontrol and could dramatically alter the benefit:cost ratio of the technology.

\section{Concluding remarks}

The successful development of biopesticide products for locust control demonstrates the potential for translation of research through to practice. Although this took several years, technical and regulatory developments should enable new applications, such as mosquito control, to advance to the market more rapidly. The specific features of fungal infection such as late acting mortality, transmission blocking and host behavioural changes, provide opportunities to minimise the risk of resistance evolution. Indeed with fungal biopesticides, we are in a perhaps unique position in malaria control history: we can think about preventing evolutionary outcomes now, rather than after a once promising method has begun to fail. There is also scope for innovative applications of established knowledge from other industries. Nonetheless, numerous research challenges remain and we need to recognise there will be technical and biological constraints that set limits to the approach. Moreover, contrasting experiences with the locust biopesticides in Africa and Australia reveal the need to support not only research and development but also, 
Thomas, M.B \& Read, A.F. 2007. Can fungal biopesticides control malaria? Nature Microbiology Reviews 5: 377-383.

implementation and capacity building; to make such technologies a reality we cannot simply rely on the initial technical innovation and market pull. This is an important interdisciplinary interpretation that sets a challenge to researchers working to develop alternative pest control technologies and the donors and agencies that support this.

Matthew B. Thomas is at CSIRO Entomology, GPO Box 1700, Canberra, ACT 2601,

Australia

Andrew F. Read is at the Institutes of Evolutionary Biology \& Immunology and Infection Research, School of Biological Sciences, University of Edinburgh, Edinburgh EH9 3JT,

$U K$

Correspondence to MBT e-mail: matthew.thomas@csiro.au

1. Hemingway, J. \& Ranson, H. Insecticide resistance in insect vectors of human disease. Annu. Rev. Entomol. 45, 369-389 (2000).

2. Zaim, M. \& Guillet, P. Alternative insecticides: an urgent need. Trends Parasitol. 18, $161-163$ (2002).

3. Hargreaves, K. et al. Anopheles arabiensis and An. quadriannulatus resistance to DDT in South Africa. Med. Vet. Entomol. 17, 417-422 (2003).

4. Thomas, M.B. \& Blanford, S. Thermal biology in insect-pathogen interactions. Trends Ecol. Evol. 18, 344-350 (2003). 
Thomas, M.B \& Read, A.F. 2007. Can fungal biopesticides control malaria? Nature Microbiology Reviews 5: 377-383.

5. Georgis, R. in Microbial Insecticides: Novelty or Necessity? (ed. Evans H.F.) 243252 (British Crop Protection Council Monograph No. 68, 1997).

6. Fravel, D.R. Commercialization and implementation of biocontrol. Annu. Rev. Phytopathol. 43, 337-359 (2005).

7. Bateman, R.P., Carey, M., Moore, D. \& Prior, C. The enhanced infectivity of Metarhizium flavoviride in oil formulations to desert locusts at low humidities. Ann. Appl. Biol. 122, 145 - 152 (1993).

8. Lomer, C.J. et al. Biological control of locusts and grasshoppers. Annu. Rev. Entomol. 46, 667 - 702 (2001).

9. Thomas, M.B., Kooyman, C. Locust biopesticides: a tale of two continents. Biocontr. News Info. 25, 47N-51N (2004).

10. Spurgin, P. Operational Use of Green Guard® for Locust and Grasshopper Control in Australia. Biocontr. News Info. 25, 51N-53N (2004).

11. Lawrence, L. A green locust control for Australian Farmers. Outlooks on Pest Management [Dec], 253-254 (2005).

12. APLC. http://www.affa.gov.au/aplc

13. Lacey, L.A. \& Undeen, A.H. Microbial control of black flies and mosquitoes. Annu. Rev. Entomol. 31, 265-296 (1986).

14. Rishikesh, N., Dubitiskij, A.M. \& Moreau in Malaria: Principles and Practices of Malariology (ed. Wernsdorfer, W.H. \& McGregor, I.) 1227-1250 (Churchill Livingstone, New York, 1988).

15. Scholte, E.-J., Knols, B.G.J., Samson, R.A. \& Takken, W. Entomopathogenic fungi for mosquito control: A review. J. Insect Sci., 4:19, 24pp (2004). 
Thomas, M.B \& Read, A.F. 2007. Can fungal biopesticides control malaria? Nature Microbiology Reviews 5: 377-383.

16. Fillinger, U. \& Lindsay, S.W. Suppression of exposure to malaria vectors by an order of magnitude using microbial larvicides in rural Kenya. Trop. Med. Int. Health 11, 1629-1642 (2006).

17. Scholte, E.-J. et al. Pathogenicity of six East African entomopathogenic fungi to adult Anopheles gambiae s.s. (Diptera: Culicidae) mosquitoes. Proc. Exp. Appl. Entomol. NEV, Amsterdam 14, $25-29$ (2003).

18. Scholte, E.-J. et al. Infection of malaria Anopheles gambiae (s.s.) and filariasis (Culex quinquefasciatus) vectors with the entomopathogenic fungus Metarhizium anisopliae. Malaria J. 2, 29 (2003).

19. Blanford, S. et al. Fungal pathogen reduces potential for malaria transmission. Science 308, 1638-1641 (2005).

20. Scholte, E-J et al. An entomopathogenic fungus for control of adult African malaria mosquitoes. Science 308, 1641-1642 (2005).

21. Scholte, E-J., Knols, B.G.J. \& Takken, W. Infection of the malaria mosquito Anopheles gambiae with the entomopathogenic fungus Metarhizium anisopliae reduces blood feeding and fecundity. J. Invertebr. Pathol. 91, 43-49 (2006).

22. Ward, M.D.W., Selgrade, M.K. Benefits and risks in malaria control. Science 310, 49 (2005).

23. Hutchinson, O.C. \& Cunningham, A.A. Benefits and risks in malaria control. Science 310, 49 (2005).

24. Thomas, M.B. et al. Benefits and risks in malaria control. Science 310, 50 (2005).

25. G. MacDonald, The epidemiology and control of malaria (Oxford University Press, London, 1957). 
Thomas, M.B \& Read, A.F. 2007. Can fungal biopesticides control malaria? Nature Microbiology Reviews 5: 377-383.

26. Anderson, R.M. in Population Biology of Infectious Diseases (ed. Anderson, R.M. \& May, R.M.) 242-261 (Chapman \& Hall, London, 1982).

27. Seyoum, E., Moore, D. \& Charnley, A.K. Reduction in flight activity and food consumption by the Desert Locust, Schistocerca gregaria, after infection with Metarhizium flavoviride. J. Appl. Entomol. 118, 310 - 315 (1994).

28. Thomas, M.B., Blanford, S., Gbongboui, C. \& Lomer, C.J. Experimental studies to evaluate spray applications of a mycoinsecticide against the rice grasshopper, Hieroglyphus daganensis, in northern Benin. Entomol. Exp. Applic. 87, 93-102 (1998).

29. Arthurs, S. \& Thomas, M.B. Effects of a mycoinsecticide on feeding and fecundity of the brown locust, Locustana pardalina. Biocontr. Sci. Technol. 10, 321-329 (2000).

30. Arthurs, S.P. \& Thomas, M.B. Investigation into behavioural changes in Schistocerca gregaria following infection with a mycoinsecticide: implications for susceptibility to predation. Ecol. Entomol. 26, 227-234 (2001).

31. Blanford, S. \& Thomas, M.B. Adult survival, maturation and reproduction of the desert locust, Schistocerca gregaria, infected with Metarhizium anisopliae var. acridum. J. Invertebr. Pathol. 78, 1-8 (2001).

32. Hargreaves, K. et al. Anopheles funestus resistant to pyrethroid insecticides in South Africa. Med. Vet. Entomol. 14, 181-189 (2000).

33. Brooke, B.D. et al. Bioassay and biochemical analyses of insecticide resistance in southern African Anopheles funestus (Diptera : Culicidae). Bull. Entomol. Res. 91, 265-272 (2001). 
Thomas, M.B \& Read, A.F. 2007. Can fungal biopesticides control malaria? Nature Microbiology Reviews 5: 377-383.

34. Hemingway, J. Taking aim at mosquitoes. Nature 430, 936 (2004).

35. Brogdon, W.G. \& McAllister, J.C. Insecticide resistance and vector control. Emerg. Infect. Dis. 4, 605-613 (1998).

36. Michalakis, Y. \& Renaud, F. Malaria - Fungal allies enlisted. Nature 435, 891. (2005).

37. Ferrari, J., Müller, C. B., Kraaijeveld, A. R. \& Godfray, H. C. J. Clonal variation and covariation in aphid resistance to parasitoids and a pathogen. Evolution 55, 18051814 (2001).

38. Blanford, S., Thomas, M.B., Pugh, C. \& Pell, J.K. Temperature checks the Red Queen? Resistance and virulence in a fluctuating environment. Ecol. Lett. 6, 2-5 (2003).

39. Tinsley, M.C., Blanford, S., Jiggins, F.M. Genetic variation in Drosophila melanogaster pathogen susceptibility. Parasitology 132, 767-773 (2006).

40. Traniello, J.F.A., Rosengaus, R.B. \& Savoie, K. The development of immunity in a social insect: Evidence for the group facilitation of disease resistance. Proc. Natl. Acad. Sci. USA 99, 6838-6842 (2002).

41. Hughes, W. O. H., Eilenberg, J. \& Boomsma, J. J. Trade-offs in group living: transmission and disease resistance in leaf-cutting ants. Proc. R. Soc. B. 269, 18111819 (2002).

42. Elliot, S.L., Blanford, S. \& Thomas, M.B. Host-pathogen interactions in a varying environment: temperature, behavioural fever and fitness. Proc. $R$. Soc. B 269, 15991607 (2002). 
Thomas, M.B \& Read, A.F. 2007. Can fungal biopesticides control malaria? Nature Microbiology Reviews 5: 377-383.

43. Partridge, L. \& Barton, N.H. Optimality, mutation and the evolution of ageing Nature 362, 305-311 (1993).

44. Boete, C. \& Koella, J.C. Evolutionary ideas about genetically manipulated mosquitoes and malaria control. Trends Parasitol. 19, 32-38 (2003).

45. Riehle, M.M. et al. Natural malaria infection in Anopheles gambiae is regulated by a single genomic control region. Science 312, 577-579 (2006).

46. Enserink, M. (2005). Microbiology - Mosquito-killing fungi may join the battle against malaria Science 308, 1531.

47. Itoh, T. Evaluation of long-lasting insecticidal nets after 2 years household use. Trop. Med. Int. Health 10, 1321-1326 (2005).

48. Maniania, N.K. A low-cost contamination device for infecting adult tsetse flies, Glossina spp., with the entomopathogenic fungus Metarhizium anisopliae in the field. Biocontr. Sci. Technol. 12, 59-66 (2002).

49. Hong, T. D., Jenkins, N.E. \& Ellis, R.H. Fluctuating temperature and the longevity of conidia of Metarhizium flavoviride in storage. Biocontr. Sci. Technol. 9, 165-176 (1999).

50. Hong, T. D., Jenkins, N.E. \& Ellis, R.H. The effects of duration of development and drying regime on the longevity of conidia of Metarhizium flavoviride. Mycol. Res. 104, 662-665 (2000).

51. Schisler, D.A. \& Slininger, P.J. Microbial selection strategies that enhance the likelihood of developing commercial biological control products. J. Ind. Microbio. Biot. 19, 172-179 (1997). 
Thomas, M.B \& Read, A.F. 2007. Can fungal biopesticides control malaria? Nature Microbiology Reviews 5: 377-383.

52. Hynes, R.K., Boyetchko, S.M. (2006). Research initiatives in the art and science of biopesticide formulations. Soil Biol. Biochem. 38, 845-849.

53. Thomas, M.B., Klass, J. \& Blanford, S. (2000). The year of the locust. Pesticide Outlook 11, 192-195.

54. Onwujekwe, O. et al. Socio-economic inequity in demand for insecticide-treated nets, in-door residual house spraying, larviciding and fogging in Sudan. Malaria J. 4, 4-62 (2005)

55. Meltzer, M.I. et al. The household-level economics of using permethrin-treated bed nets to prevent malaria in children less than five years of age. Am. J. Trop. Med. Hyg. 68, 149-160 (2003).

56. Cherry, A. J. et al. Operational and economic analysis of a West African pilot-scale production plant for aerial conidia of Metarhizium spp. for use as a mycoinsecticide against locusts and grasshoppers. Biocontr. Sci. Technol. 9, 35-51 (1999).

57. Jenkins, N.E. \& Grzywacz, D. Quality control of fungal and biocontrol agents assurance of product performance. Biocontr. Sci. Technol. 10, 753-777 (2000).

58. Sharp, B. et al. Malaria control by residual insecticide spraying in Chingola and Chililabombwe, Coperbelt Province, Zambia. Trop. Med. Int. Health 7, $732-736$ (2002).

59. WHO. Global Strategic Framework for Integrated Vector Management. WHO/CDS/CPE/PVC/2004.10. (WHO, Geneva, 2004).

60. M.A. Osta et al. Effects of mosquito genes on Plasmodium development. Science 303, 2030-2032 (2004). 
Thomas, M.B \& Read, A.F. 2007. Can fungal biopesticides control malaria? Nature Microbiology Reviews 5: 377-383.

61. Hemingway, J. \& Craig, A. Parasitology: new ways to control malaria. Science 303, 1984-1985 (2004).

62. St Leger, R.J. et al. Construction of an improved mycoinsecticide overexpressing a toxic protease. Proc. Natl. Acad. Sci. USA 93, 6349-6354 (1996).

63. Alphey, L. et al. Malaria control with genetically manipulated insect vectors. Science 298, 119-121 (2002).

64. Scott, T.W. et al. The ecology of genetically modified mosquitoes. Science 298, 117118 (2002).

65. Gillespie, J.P. et al. Fungi as elicitors of insect immune responses. Arch. Insect Biochem. Physiol. 44, 49-68 (2000).

66. Roberts, D. W. \& St. Leger, R.J. Metarhizium spp., cosmopolitan insect-pathogenic fungi: mycological aspects. Advanc. Appl. Microbiol. 54, 2-70 (2004).

\section{Acknowledgements}

Our empirical work is funded by The Wellcome Trust. We are grateful to Simon Blanford, Ken Vernick and members of the "Research Consortium for Novel and Sustainable Approaches of Adult Vector Control Based on Fungi”, particularly Maureen Coetzee, Chris Curtis, Gerry Killeen, Bart Knols and Willem Takken, for discussion and encouragement. This article was written while AR was at the Wissenshaftkolleg zu Berlin.

\section{Competing interests statement}

The authors declare no competing financial interests. 
Thomas, M.B \& Read, A.F. 2007. Can fungal biopesticides control malaria? Nature Microbiology Reviews 5: 377-383.

\section{Glossary Terms}

Appresorium Is a flattened, hyphal "pressing" organ produced by a germinating fungal spore, from which an infection peg grows and penetrates the host cuticle

Biocontrol or Biological Control is the use of living natural enemies such as predators, parasitoids or pathogens, to control pest insects, weeds, or diseases.

Biopesticide In simplest terms refers to a pesticide that is biological in origin (i.e., viruses, bacteria, fungi, pheromones, natural plant compounds). The approach is characterised by inundative applications of a living organism, with little or no reliance on the organism to reproduce or be self-sustaining in order to bring about control. In this regard, the biological control agent is being used as a chemical pesticide analogue.

Entomological Inoculation Rate (EIR) A measure of the frequency with which a human is bitten by an infectious mosquito.

Haemocoel The body cavity of an arthropod in which most of the major organs are found. It is filled with the arthropod equivalent of blood, called haemolymph.

Oocyst A walled, vegetatively replicating malarial parasite under the basal lamina of the mosquito midgut in which the transmissible sporozoites form.

Parasite incubation period The time from infection of the mosquito following a blood feed from a human host carrying malaria, to the point at which the mosquito is infectiousness and can transmit the parasite to a new host during a further feeding bout. Throughout large areas of malaria transmission the parasite incubation period is 12-14 days. 
Thomas, M.B \& Read, A.F. 2007. Can fungal biopesticides control malaria? Nature Microbiology Reviews 5: $377-383$.

Paratransgenic approaches (or Paratransgenesis) Genetic manipulation of organisms associated with a vector (usually commensal or symbiotic bacteria but in our case, fungal entomopathogens) to alter the vector's ability to transmit a pathogen. The vector itself is not genetically modified.

Sporozoite Small elongated cells resulting from repeated division of the oocyst. In the case of the malarial parasite, it is the sporozoites that concentrate in the salivary glands and are introduced into the blood by the bite of a mosquito.

Vectorial capacity Provides a measure of disease risk as determined by the ability of a vector to successfully transmit disease and incorporates aspects of vector competence, abundance, biting rates, survival rates and parasite incubation period. 
Thomas, M.B \& Read, A.F. 2007. Can fungal biopesticides control malaria? Nature Microbiology Reviews 5: 377-383.

\section{Box 1| Paratransgenic Approaches}

Whilst there is a wealth of lethal and sub-lethal properties of natural fungal isolates or isolate combinations to be explored, there would seem considerable additional promise for utilising residual sprays of fungal pathogens in novel paratransgenic approaches. Regulatory and ethical concerns notwithstanding, because fungal pathogens function by contact with the insect host they could constitute a novel delivery mechanism for antimalarial or anti-mosquito biomolecules. Genetic modification could enable fungal pathogens to express toxins or, for example, effector molecules that block sporogony within the vector ${ }^{60}$. Lack of a practical delivery mechanism has been identified as a significant constraint for malaria control interventions involving effector molecules such as $\mathrm{RNAi}^{61}$. The potential to transform fungal entomopathogens is well established. Metarhizium anisopliae, for example, has already been engineered to over-express a toxic protease to increase the speed with which is kills Lepidopteran pests in agricultural systems $^{62}$. Secondary transfer of fungi from mosquitoes is very unlikely to occur (fungal spores are only produced once the insect is dead, and many cadavers are scavenged before sporulating, limiting potential for transfer to other hosts) so that fungal transgenes would be easier to control than mosquito transgenes. Moreover, whereas encouraging the spread of transgenes in mosquito populations is fraught with ecological and population genetic problems ${ }^{63,64}$, the fitness of the transgenes (essentially the ability of a modified gene to persist and spread through a population) would be relatively unimportant in a biopesticide where repeated application is envisaged and natural reproduction and transmission are of little consequence. 
Thomas, M.B \& Read, A.F. 2007. Can fungal biopesticides control malaria? Nature Microbiology Reviews 5: 377-383.

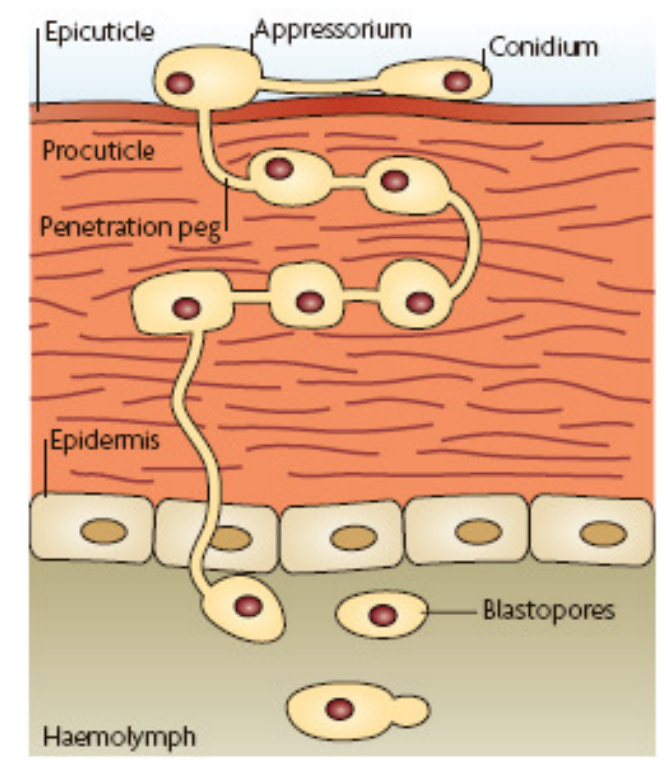

Figure 1. Fungal entomopathogens act via external contact with the insect host. The in vivo development cycle of entomopathogenic fungi, such as Beauveria bassiana and Metarhizium anisopliae, involves sequential steps of adhesion of conidia (spores) to the host cuticle, germination of the conidia and production of germ tube and appresorium (penetration structure), penetration of the cuticle via a combination of mechanical pressure and the action of cuticle degrading enzymes, vegetative growth within the host haemoceol and then production of externally-borne conidia upon death of the host ${ }^{65,66}$. The host cuticle provides the first line of defence against infection and plays a key role in determining specificity of the fungus. If the fungus breaches the cuticle, successful infection depends on subsequent ability to overcome the insect innate immune response. Insects can respond in both a cellular and humoral manner to fungal infection, with immune activation occurring as early as the point of cuticle degradation during the penetration process $^{65}$. Fungi generally have two strategies for overcoming the host defence responses; development of cryptic growth forms that are effectively masked from 
Thomas, M.B \& Read, A.F. 2007. Can fungal biopesticides control malaria? Nature Microbiology Reviews 5: 377-383.

the insect defence responses and/or production of immuno-modulating substances which suppress the defence system ${ }^{65,66}$. 
Thomas, M.B \& Read, A.F. 2007. Can fungal biopesticides control malaria? Nature Microbiology Reviews 5: $377-383$

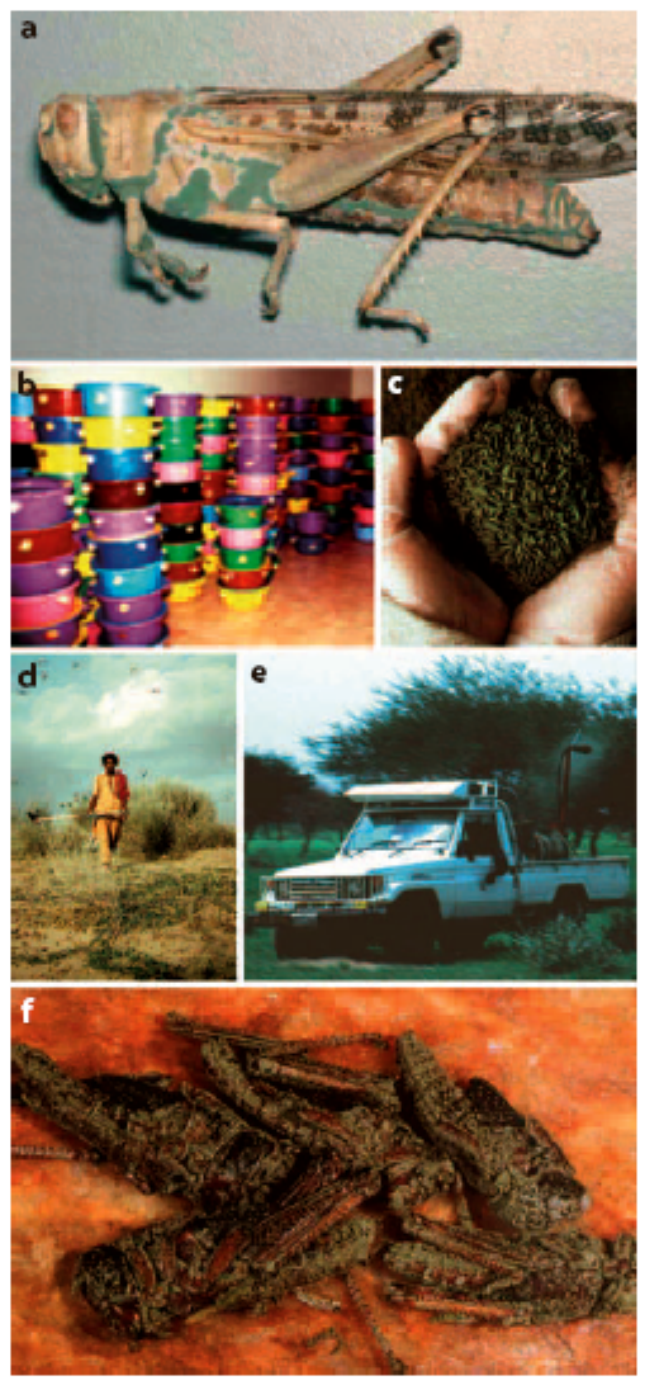

Figure 2. Use of biopesticides for sustainable control of locusts and grasshoppers in Africa. A virulent strain of the fungal entomopathogen Metarhizium anisopliae var acridum was isolated from an infected cadaver collected from the field (top picture shows a dead, infected desert locust with spores being produced at the inter-segmental membranes). Relatively simple techniques were then developed for in vitro mass production. Shown here is a small-scale production facility in west Africa where spores are grown in bowls using part-cooked rice as a solid substrate. Fungal spores are then 
Thomas, M.B \& Read, A.F. 2007. Can fungal biopesticides control malaria? Nature Microbiology Reviews 5: 377-383

harvested from the rice and formulated in oil for ultra low volume application using hand-held, vehicle or aerial-mounted (not shown) sprayers. Infected locusts and grasshoppers typically die in 7-25 days (speed of kill is strongly influenced by environmental temperature and insect thermal behaviour in this system ${ }^{4}$ ) and under conditions of high humidity produce new spores. 
Thomas, M.B \& Read, A.F. 2007. Can fungal biopesticides control malaria? Nature Microbiology Reviews 5: $377-383$.

a

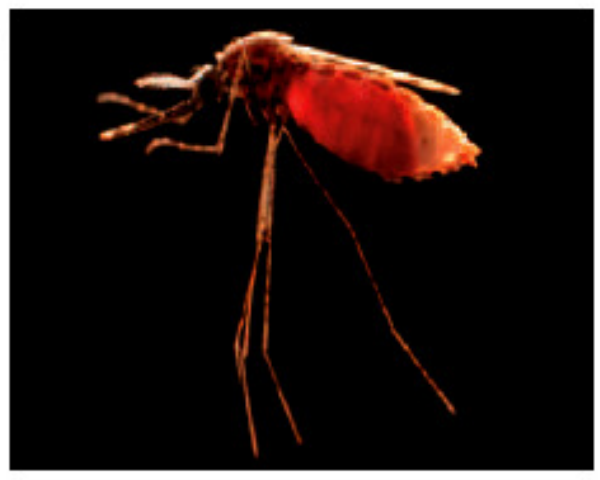

c
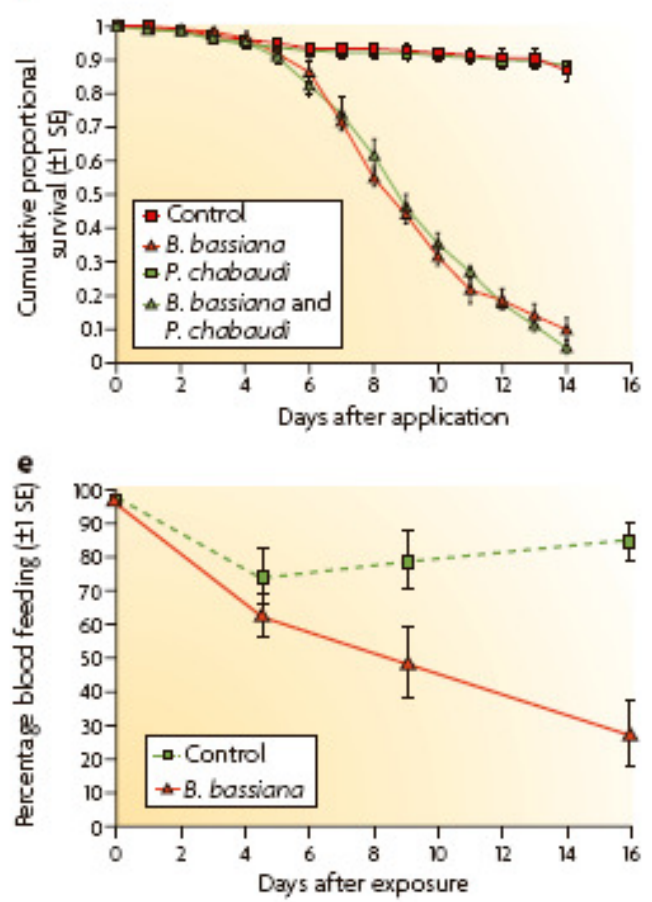

b

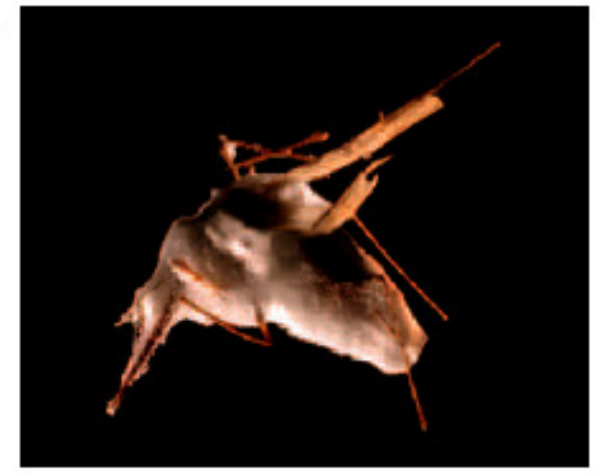

d

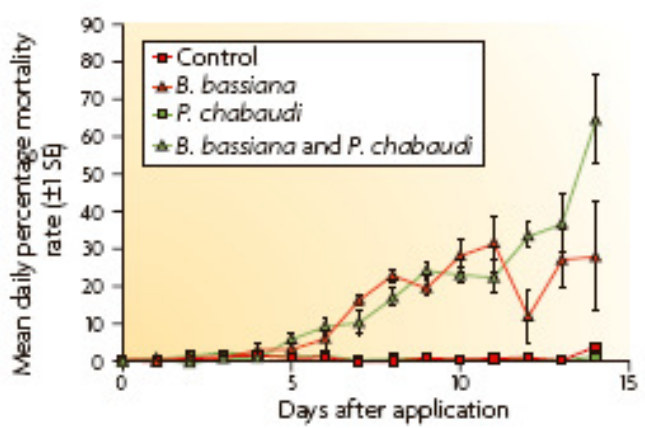

f

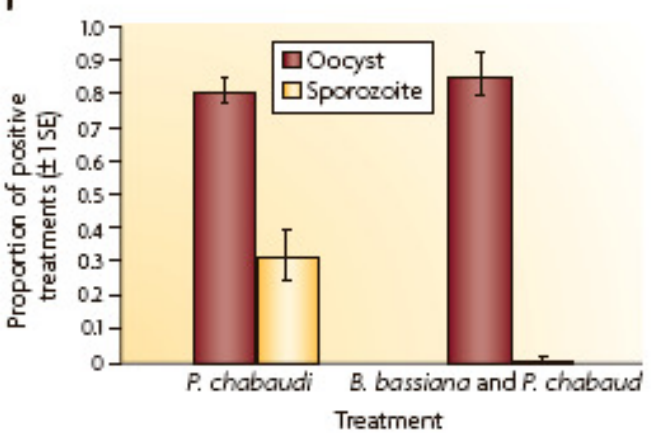

Figure 3. Fungal infection substantially reduces the proportion of mosquitoes potentially able to transmit malaria. In the fungal biopesticide approach female mosquitoes contact fungal spores from treated surfaces as they rest to digest a blood meal. As the fungal infection progresses mosquitoes die and in the right conditions eventually sporulate, producing a mat of fungal spores on the outside of the cadaver (top right). a Infection with the entomopathogenic fungus Beauveria bassiana dramatically 
Thomas, M.B \& Read, A.F. 2007. Can fungal biopesticides control malaria? Nature Microbiology Reviews 5: 377-383.

reduces survival of Anopheles stephensi mosquitoes by day 14 (the time following an infectious blood feed at which an individual mosquito becomes able to transmit malaria). b In addition there appears an interaction with malaria (Plasmodium chabaudi) whereby daily mortality rates accelerate from day 11 in those mosquitoes carrying both fungus and malaria. c Furthermore, mosquitoes infected with the fungus exhibit a significant decline in propensity to blood feed as the disease progresses. Finally, survivorship or development of the malaria parasite inside the mosquito is affected such that even if mosquitoes survive, there is less chance of having the infectious stage of the malaria parasite (the sporozoites) in their mouthparts (figure shows mean ( \pm SEM) proportion of the starting population of mosquitoes in the Malaria and Malaria+Beauveria treatments positive for malaria oocysts at day 7 and sporozoites at day 14 after infectious blood meal). The effect is an 80 -fold reduction in potential of mosquitoes to transmit malaria (from REF 19). 
Thomas, M.B \& Read, A.F. 2007. Can fungal biopesticides control malaria? Nature Microbiology Reviews 5: $377-383$.

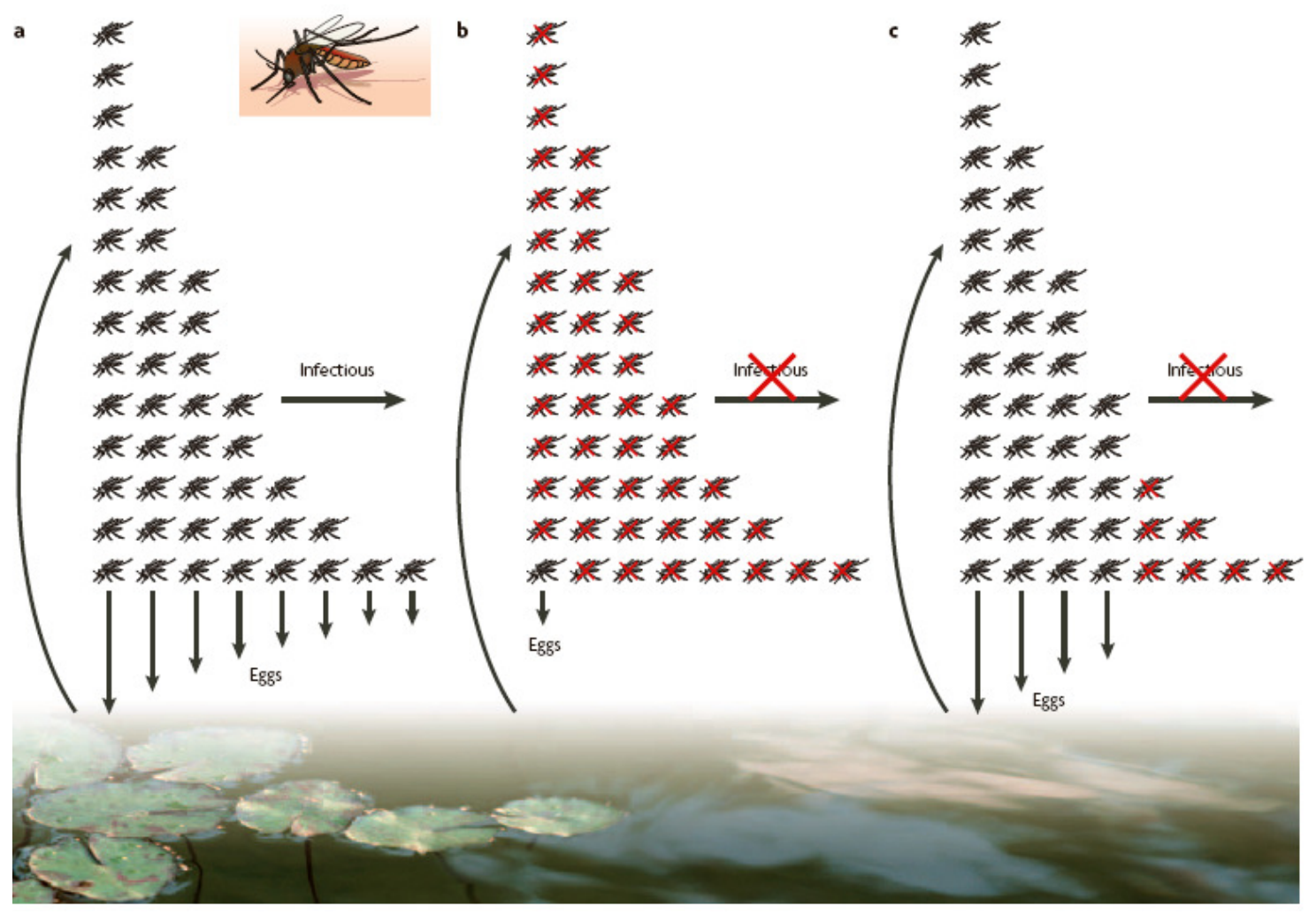

Figure 4. The sustainability of chemical and biological interventions against adult

mosquitoes. a In their normal life cycle female mosquitoes take a blood meal every 2-4 days and use this to mature sequential batches of eggs (x-axis). Natural mortality is generally high (survivors, y-axis) such that the majority of the reproductive output (vertical arrows) from a population accrues over the first 1-3 feeding/oogenic cycles. Relatively few mosquitoes actually survive long enough (12-14 days) in the field for the malaria parasite to complete its development, migrate to the mosquito mouthparts and get transmitted to a new human host ('infectious'). b Exposure to a fast acting insecticide following the first blood meal reduces survivorship and prevents malaria transmission. However, the rapid mortality carries a big fitness cost and creates a substantial selection pressure for development of resistance. $\mathbf{c}$ Relative slow speed of fungal kill helps mitigate selection pressure as infected mosquitoes can still complete the important early oogenic 
Thomas, M.B \& Read, A.F. 2007. Can fungal biopesticides control malaria? Nature Microbiology Reviews 5: 377-383.

cycles. An isolate that allows a high level of survival (and hence egg production) over the first 7-9 days, for instance, but then causes extensive mortality will still reduce malaria transmission but will impose little selection for resistance. 